\title{
Low molecular weight proteomic information distinguishes metastatic from benign pheochromocytoma
}

\author{
FM Brouwers ${ }^{1}$, E F Petricoin $I I^{2}$, L Ksinantova ${ }^{3}$, J Breza ${ }^{4}$, \\ $\checkmark$ Rajapakse ${ }^{5}$, S Ross ${ }^{5}$, D Johann ${ }^{5}$, M Mannelli,,$B$ L Shulkin', \\ $R$ Kvetnansky $^{3}, G$ Eisenhofer ${ }^{8}, M M$ Walther $^{9}, B$ A Hitt $^{10}$, \\ $T P$ Conrads $^{11}, T D$ Veenstra ${ }^{11}, D P$ Mannion ${ }^{12}, M R$ Wall $^{12}$,

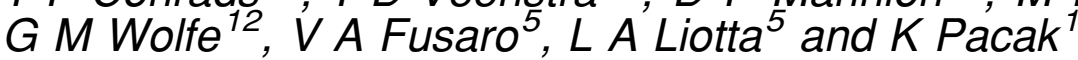

\footnotetext{
${ }^{1}$ Reproductive Biology and Medicine Branch, National Institute of Child Health and Human Development, Bethesda, Maryland, USA

${ }^{2} \mathrm{NCl} /$ FDA Clinical Proteomics Program, Office of Cell and Gene Therapies, Center for Biologics Evaluation and Research,

Food and Drug Administration, Bethesda, Maryland, USA

${ }^{3}$ Institute of Experimental Endocrinology, Slovak Academy of Sciences, Bratislava, Slovak Republic

${ }^{4}$ Department of Urology, Komensky Faculty of Medicine, Bratislava, Slovak Republic

${ }^{5} \mathrm{NCl} /$ FDA Clinical Proteomics Program, Laboratory of Pathology, Center for Cancer Research, National Cancer Institute, Bethesda, Maryland, USA

${ }^{6}$ Department of Clinical Physiopathology, Endocrine Unit, University of Florence, Florence, Italy

${ }^{7}$ Department of Radiological Sciences, St Jude Children's Research Hospital, Memphis, Tennessee, USA

${ }^{8}$ Clinical Neurocardiology Section, National Institute of Neurological Disorders and Stroke, Bethesda, Maryland, USA

${ }^{9}$ Urologic Oncology Branch, Center for Cancer Research, National Cancer Institute, National Institutes of Health, Bethesda, Maryland, USA

${ }^{10}$ Correlogic Systems Inc., Bethesda, Maryland, USA

${ }^{11}$ National Cancer Institute Biomedical Proteomics Program, Analytical Chemistry Laboratory, Mass Spectrometry Center,

SAIC-Frederick, Inc., NCI-Frederick, Frederick, Maryland, USA

${ }^{12}$ Predictive Diagnostics Inc., Vacaville, California, USA
}

(Requests for offprints should be addressed to K Pacak, Unit on Clinical Neuroendocrinology, RBMB, NIH, NICHD, Building 10, CRC, Room 1E-1-3140, 10 Center Drive, MSC-1109, Bethesda, Maryland 20892-1109,

USA; Email: karel@mail.nih.gov)

\begin{abstract}
Metastatic lesions occur in up to $36 \%$ of patients with pheochromocytoma. Currently there is no way to reliably detect or predict which patients are at risk for metastatic pheochromocytoma. Thus, the discovery of biomarkers that could distinguish patients with benign disease from those with metastatic disease would be of great clinical value. Using surface-enhanced laser desorption ionization protein chips combined with high-resolution mass spectrometry, we tested the hypothesis that pheochromocytoma pathologic states can be reflected as biomarker information within the low molecular weight (LMW) region of the serum proteome. LMW protein profiles were generated from the serum of 67 pheochromocytoma patients from four institutions and analyzed by two different bioinformatics approaches employing pattern recognition algorithms to determine if the LMW component of the circulatory proteome contains potentially useful discriminatory information. Both approaches were able to identify combinations of LMW molecules which could distinguish all metastatic from all benign pheochromocytomas in a separate blinded validation set.

In conclusion, for this study set low molecular mass biomarker information correlated with pheochromocytoma pathologic state using blinded validation. If confirmed in larger validation studies, efforts to identify the underlying diagnostic molecules by sequencing would be warranted. In the future, measurement of these biomarkers could be potentially used to improve the ability to identify patients with metastatic disease.
\end{abstract}

Endocrine-Related Cancer (2005) 12 263-272 


\section{Introduction}

Pheochromocytoma is a catecholamine-producing tumor of chromaffin cells (Pacak et al. 2001a) which can occur sporadically or as part of a familial syndrome. There are 500-1600 new cases per year in the USA (Pacak et al. 2001 b). The incidence reported for metastatic pheochromocytoma ranges (depending on the genetic background and tumor localization) from 3 to $36 \%$ of pheochromocytoma patients. Metastatic disease has an overall 5-year survival rate of 50\% (O'Riordain et al. 1996, Goldstein et al. 1999, John et al. 1999). Since there are no histological criteria for malignancy, metastatic pheochromocytoma is defined as the presence of metastatic lesions at sites where chromaffin tissue is normally absent (Linnoila et al. 1990). Metastases to lymph nodes, bones, lungs and liver are most common (Kebebew \& Duh 1998, Goldstein et al. 1999). Metastatic pheochromocytoma can manifest even 20 years after the occurrence of the primary pheochromocytoma (Tanaka et al. 1993). Currently, there is no cure for metastatic pheochromocytoma.

The lack of histological criteria for malignancy has initiated many studies to find a marker that can differentiate benign from metastatic pheochromocytoma. So far, several cancer-associated markers, such as $\mathrm{p} 53$, MIB-1 (Ki-67) and telomerase, alone or in combination, have been tested for their usefulness, but without marked success (Brown et al. 1999, de Krijger et al. 1999, Orlando \& Gelmini 2001). Furthermore, several neuroendocrine- and catecholamine-related markers such as chromogranin A and 3,4-dihydroxyphenylalanine have also been investigated (Goldstein et al. 1986, Rao et al. 2002). However, markedly elevated levels of these markers are only found in up to $60 \%$ of metastatic pheochromocytomas. Two studies have explored chromosomal abnormalities in pheochromocytoma by comparative genomic hybridization (CGH) (Dannenberg et al. 2000, Edstrom et al. 2000). Losses on chromosome $1 \mathrm{p}(82-86 \%)$ and $3 \mathrm{q}(41-52 \%)$ are seen in both benign and metastatic pheochromocytomas. In one study, $\mathrm{CGH}$ alterations on chromosome 11 appeared to be associated with metastatic pheochromocytomas (Edstrom et al. 2000), whereas in the other study deletions on chromosome $6 q$ and $17 p$ were more frequently seen in metastatic pheochromocytomas compared with benign pheochromocytomas (Dannenberg et al. 2000). Overall, the CGH alterations associated with metastatic pheochromocytoma were only seen in $50-60 \%$ (versus $21 \%$ in benign pheochromocytomas). Despite these efforts, at present there is no reliable marker or gene that can predict or detect metastatic or recurrent pheochromocytoma. Therefore all pheochromocytoma patients require life-long follow-up.

Proteomics can be defined as the global characterization and quantification of proteins and protein systems (Etzioni et al. 2003). In recent years, new developments have made proteomic technologies such as two-dimensional PAGE and mass spectrometry highly applicable to tumor biomarker discovery. For the analysis of complex protein samples mass spectrometry has evolved as the method of choice (Aebersold \& Mann 2003). Matrix-assisted laser desorption and ionization time-of-flight mass spectrometry and surfaceenhanced laser desorption ionization time-of-flight (SELDI-TOF) mass spectrometry, alone or in tandem with other proteomics techniques, have successfully identified biomarkers in diseases such as breast cancer, rheumatoid arthritis and lung cancer (Sinz et al. 2002, Vytvytska et al. 2002, Clarke et al. 2003, Rui et al. 2003). Recently, mass spectrometry-based fingerprinting has revealed the existence of previously unknown diagnostic biomarker information in the low molecular weight (LMW) region of the serum proteome (Ornstein et al. 2004, Wadsworth et al. 2004). In this study, we analyzed previously banked serum from 67 patients with pheochromocytoma, including 34 patients with metastatic disease and 33 patients with benign disease in order to determine whether LMW information existed that could differentiate between benign and malignant pathologies. For this purpose the mass spectra were analyzed by two different bioinformatics approaches, each with its own set of quality control tests. In each bioinformatics approach, the set of mass spectra that passed quality control tests (64 and 63 cases, respectively) was randomly divided into a training set and an independent validation set such that roughly an equal number of each phenotype was represented in both sets. These protein profiles were then analyzed with pattern recognition algorithms in order to identify candidate molecules with the ability to distinguish metastatic from benign pheochromocytoma with high sensitivity and specificity. Mass spectroscopy ion fingerprints that could classify the training set with the highest accuracy were selected and then separately tested using an independent set of spectra, and the results then reported.

\section{Materials and methods}

\section{Study population}

Serum samples from 67 histologically proven pheochromocytoma patients were collected at four 
participating centers that included the National Institutes of Health (NIH; Bethesda, MD, USA; 49 samples: 18 benign, 31 malignant), the University Hospital of Florence (Italy; seven samples: six benign, one malignant), the Komensky University Hospital in Bratislava (Slovak Republic; five benign samples) and the University of Michigan Medical Center (Ann Arbor, MI, USA; six samples: four benign, two malignant). All studies were done under Institutional Review Board approved protocols compliant with international guidelines. Informed consent was obtained from all patients. Patients were considered to have metastatic pheochromocytoma if pheochromocytoma tissue was found at sites where chromaffin tissue is normally absent, and had confirmatory high plasma metanephrine levels (Lenders et al. 2002). The tumor was considered to be benign if elevated metanephrine levels were found with a tumor at a site where chromaffin tissue is normally present and no evidence suggestive of metastatic disease. Samples from patients with benign and metastatic pheochromocytoma were collected according to the same protocol, during daytime clinic hours. None of the patients with metastatic disease had received any treatment specific for metastatic disease with the exception of one patient who had concluded radiation therapy 6 months prior to blood sampling.

\section{Mass spectrometry analysis}

Serum was collected according to the same protocol at all four centers. Whole blood was collected and allowed to clot at $4{ }^{\circ} \mathrm{C}$ for $30 \mathrm{~min}$ and serum was collected by centrifugation ( 3500 r.p.m. for $10 \mathrm{~min}$ ), aliquoted and stored at $-80^{\circ} \mathrm{C}$. Samples collected outside the NIH were shipped to the NIH and stored and processed in a fashion similar to those samples collected at NIH.

High resolution spectrometry and pattern recognition data analysis were performed as previously described (Petricoin et al. 2002 a, Conrads et al. 2004). All samples were run, randomized and commingled at the same time on the same instrument to minimize impact from potential sample bias. Briefly, thawed unfractionated serum samples were applied in randomly selected spots on a weak cation exchange protein chip (Ciphergen Biosystems, Freemont, CA, USA), and subjected to SELDI-TOF mass spectrometry on a QSTAR Pulsar $i$ (Applied Biosystems Inc., Framingham, MA, USA). Data were collected without filters or reduced using a priori peak picking software. The data streams for the high-resolution spectra (QSTAR, Applied Biosystems Inc.) were first binned using a function of 400 parts per million. The binning process condenses the number of data points from 350000 to exactly 7084 points per sample. To perform spectral quality control (QC) and quality assurance (QA), raw and binned data were subjected to plotting by total ion current (total record count), average/mean and standard deviation of amplitude, chi-square and $t$-test analysis of each ion or bin, and quartile plotting measures using JMP software (SAS Institute, Cary, NC, USA) as well as stored procedures developed in-house. Process measures were checked by analyzing the statistical plots of the serum reference standard (SRM-015A; National Institutes of Standards and Technology, Gaithersburg, MO, USA) that applied at random points on each chip at different spot locations.

The resultant spectral data streams were exported as '.csv' files and analyzed by two different types of bioinformatics systems: an artificial intelligence-type pattern recognition algorithm (Proteome Quest beta version 1.0; Correlogic Systems Inc., Bethesda, MD, USA) and by Bio-marker AMplifier/Filter (BAMF), a pattern recognition discovery platform (Predictive Diagnostics, Inc., Vacaville, CA, USA). After performing QC and QA measures of the spectra, spectra that failed statistical checks for homogeneity were eliminated from in-depth modelling and analysis (Conrads et al. 2004). Therefore, in the present study, three samples were excluded from analysis by Proteome Quest (all three patients with metastatic pheochromocytoma, two samples from NIH and one from the University of Michigan Medical Center) and four by BAMF (the same three samples excluded by Proteome Quest plus one benign sample in addition (case 10 in Table 2), also from the University of Michigan Medical Center). Characteristics of the study population and clinical details are listed in Tables 1 and 2.

Both bioinformatics approaches employ pattern recognition analyses on the resultant spectra in two phases: (I) training with randomly chosen known serum samples and (II) validation with a test set of blinded samples that had not been used in the training set. For example, in phase I, Proteome Quest's genetic algorithm attempts to identify, through a neo-Darwinistic 'survival of the fittest' approach, a limited number of clusters in $\mathrm{N}$-dimensional space. The clusters are plots of the Euclidean distance vector, comprised of the combined normalized intensities of the randomly sampled $\mathrm{m} / \mathrm{z}$ values of the cases and controls. In phase II, masked test spectra are then analyzed and the distance vector calculated and plotted for each sample using only the $\mathrm{m} / \mathrm{z}$ species comprising the diagnostic model identified in training. $\mathrm{N}$-dimensional plotting yields classification as disease, control, or neither, depending on whether the sample falls into previously existing case or control clusters formed in training, or establishes a 
Table 1 Study population characteristics

\begin{tabular}{|c|c|c|c|}
\hline & \multirow{2}{*}{$\begin{array}{c}\text { Total } \\
\text { Patients }\end{array}$} & \multicolumn{2}{|c|}{ Clinical behavior } \\
\hline & & Benign & Metastatic \\
\hline Number & 64 & 33 & 31 \\
\hline $\begin{array}{l}\text { Gender } \\
\text { Age (years) }\end{array}$ & $31 \mathrm{M} / 33 \mathrm{~F}$ & $14 \mathrm{M} / 19 \mathrm{~F}^{*}$ & $17 \mathrm{M} / 14 \mathrm{~F}$ \\
\hline Median & 41 & 39 & 43 \\
\hline Range & $10-84$ & $10-82$ & $21-84$ \\
\hline $\begin{array}{l}\text { Average } \pm \text { S.D. } \\
\text { Genetic } \\
\text { background }\end{array}$ & $41.1 \pm 16.4$ & $40.3 \pm 17.5$ & $41.9 \pm 15$ \\
\hline Sporadic & & 19 & 29 \\
\hline MEN2A & & 7 & 2 \\
\hline VHL & & 5 & \\
\hline Other familial & & 2 & \\
\hline
\end{tabular}

MEN2A, multiple endocrine neoplasia 2A; VHL, van Hippel Lindan syndrome.

*One sample of a female patient with benign pheochromocytoma was excluded from the analysis employing BAMF as the mass spectrum failed the quality control tests.

new cluster. Exact $95 \%$ confidence intervals (CI) were constructed for the sensitivity and specificity results from the SELDI-TOF data.

The BAMF platform comprises three process steps in the learning mode to train the system and provide the framework for analyzing data and providing results based on specific inputs. These steps include: Outlier Rejection (also known as 'Pre-Processing'), Feature Discovery and Model Building. In classification mode, the BAMF platform comprises the process steps of Outlier Rejection and Categorization. The Pre-Processing comprises quality control steps used to identify and remove spectra represented by systemic error and to ensure an acceptable level of uniformity of the data. Multiple methods of Feature Discovery provide a level of corroboration over the validity of suspect features. Model Building and Categorization combine the feature selection algorithms with multiple independent categorizers. The Model Building process is used to build and test all the possible models of the identified biomarkers to determine which biomarkers in which combinations have the best diagnostic utility. Irrespective of the approach used, all classification algorithms that represent the Model Building step undergo a consensus function for determination of the final selected class of categorizers.

Once the BAMF platform has built a suitable model, data from unknown samples may be input. The BAMF system will then perform Outlier Rejection as explained above and Categorization of the data against the model. Categorization in diagnostic (or test) mode is achieved by using at least two categorizing algorithms to categorize a sample as falling within a particular group. In addition, consensus analysis of the output of at least two categorizers is used to bolster the performance of the Categorization and to produce a confidence level for the categorization.

To generate models to distinguish pheochromocytoma patients with benign disease from those with metastatic disease Proteome Quest included 17-20 patients with benign pheochromocytoma and 15-19 with metastatic pheochromocytoma in the training set. The validation set consisted of samples of 13-16 benign and 12-16 metastatic pheochromocytoma patients.

In the analysis by BAMF, the training set comprised 21 samples of patients with benign pheochromocytoma and 21 samples of patients with metastatic pheochromocytoma. The validation set consisted of a separate set of 21 unknown samples (11 benign and 10 metastatic pheochromocytoma samples).

The full set of the raw mass spectra used in this study will be posted and available for download and analysis at: http://home.ccr.cancer.gov/ncifdaproteomics/.

\section{Results}

Both Proteome Quest and BAMF were able to identify ions with classifying potential indicating that the LMW region contained a source of information that can differentiate between pheochromocytoma patients with metastatic disease from those with benign disease.

Proteome Quest generated four models that all correctly classified all metastatic (models $1-3: n=16$, model 4: $n=12$ ) from benign samples (models 1-3: $n=16$, model 4: $n=13$ ) in the analysis of spectra of the masked validation set. The results of these models yielded $100 \%$ specificity $(16 / 16$ in models $1-3 ; 95 \%$ CI $79.4-100 \%$ and $12 / 12$ in model $4 ; 95 \%$ CI $75-100 \%$ ) and $100 \%$ sensitivity (models $1-3: 95 \%$ CI $79.4-100 \%$, model 4: 95\% CI 73-100\%) (Fig. 1).

While each of the models used separate combinations of features to attain $100 \%$ accuracy, there were several features that were found consistently (m/z 2910, 3953-56, 6681 and 9314) in several models, which may indicate their importance as prominent discriminatory objects (highlighted in Fig. 1). In fact, separate regions of the same peak were found independently in different training runs, which could indicate a higher level of importance of the contribution of this peak to the classifier pattern.

BAMF generated one model to differentiate benign from metastatic pheochromocytoma with $100 \%$ sensitivity and specificity based on the ion amplitude pattern at 6 key ions: 3894.41, 4072.71, 6071.11, 6403.01, 7193.41 and 8126.81 (Fig. 2). 
Table 2. Patient characteristics (A, asian; B, black; $H$, hispanic and $W$, white)

\begin{tabular}{|c|c|c|c|c|c|c|}
\hline Case & Sex & $\begin{array}{c}\text { Age } \\
\text { (years) }\end{array}$ & Race & Tumor behavior & Category & Tumor location \\
\hline 1 & $\mathrm{M}$ & 23 & W & Benign & Sporadic & Adrenal \\
\hline 2 & $M$ & 23 & W & Benign & Sporadic & Adrenal \\
\hline 3 & $M$ & 82 & W & Benign & Sporadic & Adrenal \\
\hline 4 & $M$ & 81 & W & Benign & Sporadic & Adrenal \\
\hline 5 & $M$ & 12 & W & Benign & Sporadic & Adrenal \\
\hline 6 & $\mathrm{M}$ & 28 & W & Benign & Sporadic & Adrenal \\
\hline 7 & $M$ & 54 & W & Benign & Sporadic & Adrenal \\
\hline 8 & M & 65 & W & Benign & Sporadic & Extra-adrenal \\
\hline 9 & $M$ & 33 & $A$ & Benign & Sporadic & Adrenal \\
\hline 10 & $\mathrm{~F}$ & 41 & W & Benign & Sporadic & Adrenal \\
\hline 11 & $\mathrm{~F}$ & 55 & $B$ & Benign & Sporadic & Adrenal \\
\hline 12 & $\mathrm{~F}$ & 54 & W & Benign & Sporadic & Adrenal \\
\hline 13 & $\mathrm{~F}$ & 54 & W & Benign & Sporadic & Adrenal \\
\hline 14 & $\mathrm{~F}$ & 43 & W & Benign & Sporadic & Adrenal \\
\hline 15 & $\mathrm{~F}$ & 57 & W & Benign & Sporadic & Adrenal \\
\hline 16 & $\mathrm{~F}$ & 43 & W & Benign & Sporadic & Extra-adrenal \\
\hline 17 & $\mathrm{~F}$ & 53 & W & Benign & Sporadic & Adrenal \\
\hline 18 & $\mathrm{~F}$ & 23 & W & Benign & Sporadic & Adrenal \\
\hline 19 & $\mathrm{~F}$ & 63 & W & Benign & Sporadic & Adrenal \\
\hline 20 & M & 33 & W & Benign & MEN2A & Adrenal \\
\hline 21 & $\mathrm{~F}$ & 29 & W & Benign & MEN2A & Adrenal \\
\hline 22 & M & 43 & W & Benign & MEN2A & Bilateral adrenal \\
\hline 23 & $M$ & 41 & $A$ & Benign & MEN2A & Bilateral adrenal \\
\hline 24 & $\mathrm{~F}$ & 32 & W & Benign & MEN2A & Adrenal \\
\hline 25 & $\mathrm{~F}$ & 39 & W & Benign & MEN2A & Extra-adrenal \\
\hline 26 & $\mathrm{~F}$ & 33 & W & Benign & MEN2A & Adrenal \\
\hline 27 & M & 20 & W & Benign & VHL & Adrenal \\
\hline 28 & $M$ & 39 & W & Benign & VHL & Adrenal \\
\hline 29 & $\mathrm{~F}$ & 45 & W & Benign & VHL & Bilateral adrenal \\
\hline 30 & $\mathrm{~F}$ & 10 & W & Benign & VHL & Extra-adrenal \\
\hline 31 & $\mathrm{~F}$ & 18 & W & Benign & VHL & Extra-adrenal \\
\hline 32 & $\mathrm{~F}$ & 25 & W & Benign & Familial & Bilateral adrenal \\
\hline 33 & $\mathrm{~F}$ & 36 & W & Benign & Familial & Extra-adrenal \\
\hline 34 & M & 23 & W & Metastatic & Sporadic & Bladder \\
\hline 35 & $M$ & 39 & W & Metastatic & Sporadic & Bone \\
\hline 36 & M & 30 & W & Metastatic & Sporadic & Lung, liver \\
\hline 37 & $M$ & 57 & W & Metastatic & Sporadic & Bone \\
\hline 38 & M & 45 & W & Metastatic & Sporadic & Liver, aortic arch \\
\hline 39 & M & 27 & $\mathrm{H}$ & Metastatic & Sporadic & Lung, abdomen, neck \\
\hline 40 & M & 21 & W & Metastatic & Sporadic & Prostate, bladder, bone \\
\hline 41 & M & 24 & W & Metastatic & Sporadic & Abdomen \\
\hline 42 & M & 84 & W & Metastatic & Sporadic & Kidney, retroperitoneal \\
\hline 43 & M & 70 & W & Metastatic & Sporadic & Lung, retroperitoneal \\
\hline 44 & M & 24 & $A$ & Metastatic & Sporadic & Lung, retroperitoneal, bone \\
\hline 45 & M & 32 & W & Metastatic & Sporadic & Retroperitoneal \\
\hline 46 & M & 56 & W & Metastatic & Sporadic & Lymph nodes \\
\hline 47 & M & 47 & $A$ & Metastatic & Sporadic & Lymph nodes \\
\hline 48 & M & 54 & $\mathrm{~B}$ & Metastatic & Sporadic & Bone, liver, lung \\
\hline 49 & $M$ & 26 & W & Metastatic & Sporadic & Liver, bone \\
\hline 50 & $\mathrm{~F}$ & 53 & W & Metastatic & Sporadic & Liver, pancreas \\
\hline 51 & $\mathrm{~F}$ & 44 & W & Metastatic & Sporadic & Retroperitoneal \\
\hline 52 & $\mathrm{~F}$ & 49 & W & Metastatic & Sporadic & Lung, neck, liver, kidney \\
\hline 53 & $\mathrm{~F}$ & 49 & W & Metastatic & Sporadic & Liver, bone \\
\hline 54 & $\mathrm{~F}$ & 27 & W & Metastatic & Sporadic & Lung, bone \\
\hline 55 & $\mathrm{~F}$ & 44 & $\mathrm{H}$ & Metastatic & Sporadic & Liver, bone \\
\hline 56 & $\mathrm{~F}$ & 32 & $\mathrm{H}$ & Metastatic & Sporadic & Liver, kidney, lymph nodes \\
\hline 57 & $\mathrm{~F}$ & 21 & W & Metastatic & Sporadic & Spine \\
\hline 58 & $\mathrm{~F}$ & 59 & W & Metastatic & Sporadic & Abdomen, liver \\
\hline 59 & $\mathrm{~F}$ & 42 & W & Metastatic & Sporadic & Liver \\
\hline 60 & $\mathrm{~F}$ & 39 & A & Metastatic & Sporadic & Liver \\
\hline 61 & $\mathrm{~F}$ & 53 & W & Metastatic & Sporadic & Soft tissue abdomen \\
\hline 62 & $\mathrm{~F}$ & 46 & W & Metastatic & Sporadic & Spleen, soft tissue abdomen \\
\hline 63 & $\mathrm{~F}$ & 39 & W & Metastatic & MEN2A & Liver, bone \\
\hline 64 & M & 43 & W & Metastatic & MEN2A & Retroperitoneal \\
\hline
\end{tabular}




\begin{tabular}{|c|c|c|c|c|c|}
\hline Model 1 & & & Model 2 & & \\
\hline Included in analysis: & Training & Validation & Included in analysis: & Training & Validation \\
\hline Benign & 17 & 16 & Benign & 17 & 16 \\
\hline Metastatic & 15 & 16 & Metastatic & 15 & 16 \\
\hline Results Validation: & & & Results Validation: & & \\
\hline Sensitivity $\quad 100 \%$ & & & Sensitivity $\quad 100 \%$ & & \\
\hline Specificity $\quad 100 \%$ & & & Specificity $\quad 100 \%$ & & \\
\hline Predicted / Actual & Benign & Metastatic & Predicted / Actual & Benign & Metastatic \\
\hline Benign & 16 & 0 & Benign & 16 & 0 \\
\hline Metastatic & 0 & 16 & Metastatic & 0 & 16 \\
\hline Model summary: & Benion & Metastatic & Model summary: & Benion & Metastatic \\
\hline Clusters & 1 & 4 & Clusters & 2 & 3 \\
\hline Key ions $(\mathrm{m} / \mathrm{z})$ & & & Key ions $(\mathrm{m} / \mathrm{z})$ & & \\
\hline 1. 1747.7715 & 5.4005 .932 & & 1. 1450.023 & 5.3956 .575 & \\
\hline 2. 3543.0955 & 6. 6429.399 & & 2. 1866.245 & 6. 6681.039 & \\
\hline 3. 3910.9526 & 7. 6681.0386 & & 3. 2214.648 & 7.9314.814 & \\
\hline 4. 3953.411 & 8.9314 .814 & & 4. 3217.55 & 8.9629 .194 & \\
\hline Model 3 & & & Model 4 & & \\
\hline$\underline{\text { Included in analysis: }}$ & Training & Validation & Included in analysis: & Training & Validation \\
\hline Benign & 17 & 16 & Benign & 20 & 13 \\
\hline Metastatic & 15 & 16 & Metastatic & 19 & 12 \\
\hline Results Validation: & & & Results Validation: & & \\
\hline Sensitivity $\quad 100 \%$ & & & Sensitivity $\quad 100 \%$ & & \\
\hline Specificity $\quad 100 \%$ & & & Specificity $\quad 100 \%$ & & \\
\hline Predicted / Actual & Benign & Metastatic & Predicted / Actual & Benign & Metastatic \\
\hline Benign & 16 & 0 & Benign & 13 & 0 \\
\hline Metastatic & 0 & 16 & Metastatic & 0 & 12 \\
\hline Model summary: & Benign & Metastatic & $\underline{\text { Model summary: }}$ & Benign & Metastatic \\
\hline Clusters & 2 & 4 & Clusters & 3 & 5 \\
\hline Key ions $(\mathrm{m} / \mathrm{z})$ & & & Key ions $(\mathrm{m} / \mathrm{z})$ & & \\
\hline 1. 899.8517 & 5. 3498.042 & & 1.887 .6989 & 5.6421 .69 & \\
\hline 2. 1554.517 & 6.4167 .716 & & 2. 1494.771 & 6. 6483.623 & \\
\hline 3.2910 .25 & 7.6681 .039 & & 3. 2911.414 & 7. 7151.053 & \\
\hline 4. 3315.519 & 8. 6761.677 & & $4 . \mathbf{5 8 1 5 . 3 5 4}$ & 8.9404 .649 & \\
\hline
\end{tabular}

Figure 1 Proteome Quest generated four separate models that could discriminate $100 \%$ of all benign and metastatic samples. The number of samples used in training and testing is shown along with the validation results, number of clusters formed that contained a majority of benign or metastatic patients and the features that underpin each model. Additionally, those features that were found to be reproducibly attained between the models are highlighted.

\section{Discussion}

In the present study we evaluated the hypothesis that the LMW region of the serum proteome contains information based on which pheochromocytoma patients with metastatic disease can be discriminated from those with benign disease. We found that two independent bioinformatics tools both correctly classified all metastatic pheochromocytomas and distinguished these from all of the patients with benign pheochromocytomas in a masked validation set. Thus, based on these initial results, proteomic fingerprinting analysis appears to point to the existence of potential diagnostic information which can then be used as a tool to distinguish between patients with benign and metastatic pheochromocytoma.

Currently, proteomics is evolving as a valuable approach in the detection and staging of many diseases, in particular cancer (Aebersold \& Mann, 2003, Wulfkuhle et al. 2003). The LMW region of the serum 


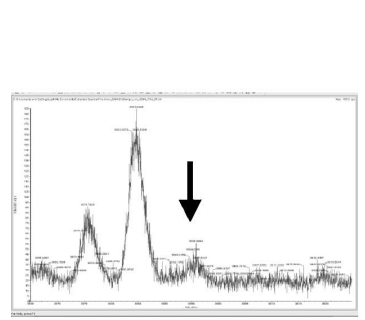

$\mathrm{m} / \mathrm{z} 3894.41$

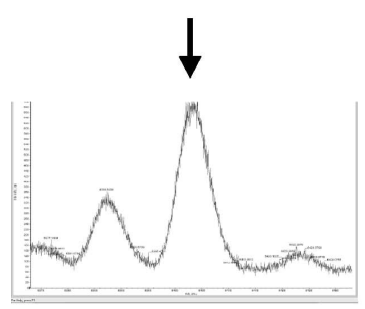

$\mathrm{m} / \mathrm{z} 6403.01$

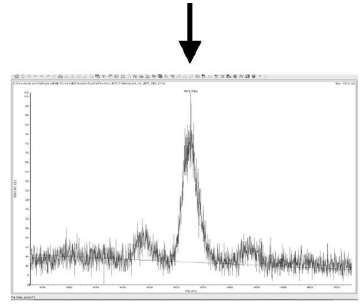

$\mathrm{m} / \mathrm{z} 4072.71$

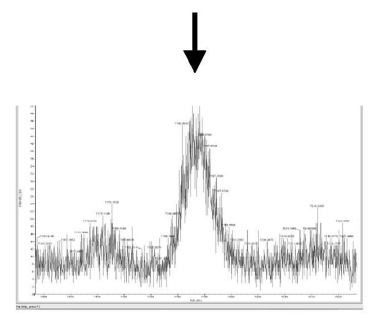

$\mathrm{m} / \mathrm{z} 7193.41$

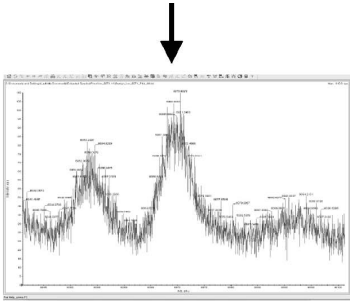

$\mathrm{m} / \mathrm{z} 6071.11$

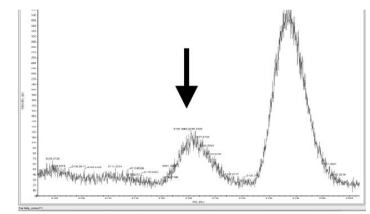

$\mathrm{m} / \mathrm{z} 8126.81$

Figure 2 Representative magnified spectral images of each of the classifying ion peaks identified by the BAMF algorithm. Ions at $\mathrm{m} / \mathrm{z}=3894.41,4072.71,6071.11,6403.01,7193.41$ and 8126.81 are shown from representative patients with benign, metastatic, benign, metastatic, metastatic and metastatic pheochromocytoma respectively. The classifying ion is marked with an arrow.

and plasma proteome, the region effectively analyzed by mass spectral analysis, contains an unexplored repository of peptides and protein fragments that could contain important diagnostic information (Liotta et al. 2003, Mehta et al. 2003). This information archive has been recently discovered to exist in a bound state complexed with high abundance circulating carrier proteins such as albumin that protect these small molecular weight biomarkers from kidney clearance and act as an amplification reagent (Liotta et al. 2003, Mehta et al. 2003). This archive can be efficiently profiled by mass spectrometry in a small starting quantity of body fluids with a high throughput non-biased discovery approach using rapid and cost-effective methods. Serum proteomic fingerprinting approaches have successfully identified potential biomarkers associated with breast cancer (Le Naour et al. 2001, Rui et al. 2003) and effectively detected ovarian cancer (Petricoin et al. 2002a), prostate cancer (Adam et al. 2002, Petricoin et al. 2002 b, Qu et al.2002), pancreatic cancer (Hingorani et al. 2003) and different stages of breast cancer (Li et al. 2002). This approach, however, has never been applied to characterize neural crest tumors, including pheochromocytoma.

In the present study we have included 64 patients with pheochromocytoma. Compared with other studies using the same methods our group of patients was smaller, but it should be noted that (a) pheochromocytoma is a rare tumor with only $500-1600$ new cases per year in the USA (Pacak et al. 2001b) and (b) we validated our results using an entirely blinded testing set. Using appropriate statistical and mathematical methods, we were able to distinguish patients with benign tumors from patients with metastatic tumors with $100 \%$ sensitivity and specificity. It is important to emphasize that the samples used in both training and blinded testing were collected at four different institutes, thus these discriminatory fingerprints may be able to transcend institute heterogeneity.

In fact, this is one of the first reported studies in which serum proteomic profiling has been employed in which patient serum samples, collected at multiple institutes, have been analyzed. For a rare tumor like pheochromocytoma a multiple center approach is crucial to obtain a sufficient sample population. Possible pitfalls of such an approach include the risk that the bioinformatics analysis programs identify differences in mass spectral signals that are the result of differences between institutes in sample handling and storage. These features are thus not based on biological differences between samples. To minimize these effects, serum samples were collected at all participating institutes according to the same protocol, and were run at the same time on the same mass spectrometer. Moreover, samples from each institute were used in training with the hope that differences associated with biology and any institute collection bias would be minimized. The fact that the bioinformatics tools could be trained with small numbers in the training sets that generated 
models with high sensitivity and specificity suggests that the study set was fairly homogenous and that the biological differences dominated influences introduced by multi-center sampling, and appear to transcend institute variability. An advantage of this approach is that the results thus obtained closely resemble a possible future clinical setting, where patients are seen at different institutes for sample collection, and specimens are then shipped out to be tested by dedicated laboratories.

Upon expansion of the study population, it is probable that sensitivity and specificity may decrease even though we used a blinded testing set as a means of evaluating the utility of the fingerprints found. However, these results remain remarkable since there is currently no reliable marker that can distinguish between metastatic and benign pheochromocytoma. Moreover, since we have multiple equally wellperforming models, the possibility of one or a collection of the ions within the patterns that continue to perform well in further validation increases. Within the four successful ion pattern fingerprints generated by Proteome Quest, some features were found repetitively. There was no overlap in key features between the model generated by BAMF and those by Proteome Quest. However, from a mathematical perspective it is a logical consequence of the complexity of the information content analyzed that multiple combinations of diagnostic features can be generated from the same starting data when employing different analysis tools (Grizzle et al. 2004). The fact that multiple patterns exist, and each uses different combinations of markers, points to the potential untapped complexity of many good diagnostic markers in this region of the blood proteome. Efforts to identify the LMW proteins/ peptides will be made after further validation and analysis.

The search for biomarkers in pheochromocytoma is hindered by the fact that patients classified as benign pheochromocytoma may develop metastases up to 20 years after the occurrence of the primary tumor (Tanaka et al. 1993). It cannot be excluded that some patients currently classified as benign pheochromocytoma will manifest with metastatic pheochromocytoma in the years to come. At that time the program can be retrained based on the new classification of these patients and this, together with an expansion of the patient population, will strengthen the reliability of the analysis. After proper validation in prospective cohort studies the measurement of these biomarkers might ultimately be used in clinical practice together with other markers for metastatic pheochromocytoma, so that the combined positive-predictive value will be high enough to prevent high numbers of false positives. Since several of the features found were identified in multiple models, we can now use this information to triage which fragments to identify first. Even after identification, it is possible that a diagnostic test would still rely on mass spectrometry to record the presence of the fragment since the development of a specific antibody that recognizes the diagnostically important fragment and not the parental molecule may prove impossible.

Our data indicate that serum-borne LMW molecules may hold great promise to characterize metastatic pheochromocytoma. The clinical impact of these results is important since there is a potential to detect patients with metastatic pheochromocytoma in an early stage and to present them with appropriate follow-up and treatment. Whether measurement of the classifying LMW information performed using direct mass spectrometry-based protein profiling, a multiplexed immunoassay panel, or a hybrid of each can be used to predict a metastatic pheochromocytoma or the potential to develop metastases in the future in patients with so called benign pheochromocytoma is unknown and cannot be concluded from this study.

The retrospective study reported herein is based on the collection of serum samples from patients who subsequently underwent an invasive procedure rendering a definitive diagnosis. While not prospective, the study does support the hypothesis that novel LMW biomarkers exist in the serum and could be useful for improved stratification of metastatic from benign disease if validated in large study sets. Efforts to sequence and identify the ions themselves would be warranted if further validation proves successful. Risk stratification-type applications could have a dramatic clinical impact both on an economic front and the reduction of morbidity and mortality. The high specificity and sensitivity found in this study indicate that an LMW information archive may contain a rich and untapped source for biomarker discovery that warrants further investigation, evaluation and rigorous validation.

\section{Funding}

No financial support was received for this study. The authors declare that there is no conflict of interest that would prejudice the impartiality of this scientific work.

\section{References}

Adam BL, Qu Y, Davis JW, Ward MD, Clements MA, Cazares LH, Semmes OJ, Schellhammer PF, Yasui Y, Feng Z et al. 2002 Serum protein fingerprinting coupled 
with a pattern-matching algorithm distinguishes prostate cancer from benign prostate hyperplasia and healthy men. Cancer Research 62 3609-3614.

Aebersold R \& Mann M 2003 Mass spectrometry-based proteomics. Nature 422 198-207.

Brown HM, Komorowski RA, Wilson SD, Demeure MJ \& Zhu YR 1999 Predicting metastasis of pheochromocytomas using DNA flow cytometry and immunohistochemical markers of cell proliferation: a positive correlation between MIB-1 staining and malignant tumor behavior. Cancer 86 1583-1589.

Clarke W, Silverman BC, Zhang Z, Chan DW, Klein AS \& Molmenti EP 2003 Characterization of renal allograft rejection by urinary proteomic analysis. Annals of Surgery 237 660-664.

Conrads TP, Fusaro VA, Ross S, Johann D, Rajapakse V, Hitt BA, Steinberg SM, Kohn EC, Fishman DA, Whitely $\mathrm{G}$ et al. 2004 High-resolution serum proteomic features for ovarian cancer detection. Endocrine-Related Cancer 11 163-178.

Dannenberg H, Speel EJ, Zhao J, Saremaslani P, van Der Harst E, Roth J, Heitz PU, Bonjer HJ, Dinjens WN, Mooi WJ et al. 2000 Losses of chromosomes $1 p$ and $3 q$ are early genetic events in the development of sporadic pheochromocytomas. American Journal of Pathology 157 353-359.

Edstrom E, Mahlamaki E, Nord B, Kjellman M, Karhu R, Hoog A, Goncharov N, Teh BT, Backdahl M \& Larsson C 2000 Comparative genomic hybridization reveals frequent losses of chromosomes $1 p$ and $3 q$ in pheochromocytomas and abdominal paragangliomas, suggesting a common genetic etiology. American Journal of Pathology 156 651-659.

Etzioni R, Urban N, Ramsey S, McIntosh M, Schwartz S, Reid B, Radich J, Anderson G \& Hartwell L 2003 The case for early detection. Nature Reviews Cancer 3 243-252.

Goldstein DS, Stull R, Eisenhofer G, Sisson JC, Weder A, Averbuch SD \& Keiser HR 1986 Plasma 3,4dihydroxyphenylalanine (dopa) and catecholamines in neuroblastoma or pheochromocytoma. Annals of Internal Medicine 105 887-888.

Goldstein RE, O'Neill JA Jr, Holcomb GW 3rd, Morgan WM 3rd, Neblett WW 3rd, Oates JA, Brown N, Nadeau J, Smith B, Page DL et al. 1999 Clinical experience over 48 years with pheochromocytoma. Annals of Surgery 229 755-764.

Grizzle WE \& Meleth S 2004 Clarification in the point/ counterpoint discussion related to surface-enhanced laser desorption/ionization time-of-flight mass spectrometric identification of patients with adenocarcinomas of the prostate. Clinical Chemistry 50 1475-1477.

Hingorani SR, Petricoin III EF, Maitra A, Rajapakse V, King C, Jacobetz MA, Ross S, Conrads TP, Veenstra TD, Hitt BA et al. 2003 Preinvasive and invasive ductal pancreatic cancer and its early detection in the mouse. Cancer Cell 4 437-450.
John H, Ziegler WH, Hauri D \& Jaeger P 1999 Pheochromocytomas: can malignant potential be predicted? Urology 53 679-683.

Kebebew E \& Duh QY 1998 Benign and malignant pheochromocytoma: diagnosis, treatment, and followup. Surgical and Oncology Clinics of North America 7 765-789.

de Krijger RR, van der Harst E, van der Ham F, Stijnen T, Dinjens WN, Koper JW, Bruining HA, Lamberts SW \& Bosman FT 1999 Prognostic value of p53, bcl-2, and cerbB-2 protein expression in phaeochromocytomas. Journal of Pathology 188 51-55.

Le Naour F, Misek DE, Krause MC, Deneux L, Giordano TJ, Scholl S \& Hanash SM 2001 Proteomics-based identification of RS/DJ-1 as a novel circulating tumor antigen in breast cancer. Clinical Cancer Research 7 3328-3335.

Lenders JW, Pacak K, Walther MM, Linehan WM, Mannelli M, Friberg P, Keiser HR, Goldstein DS \& Eisenhofer G 2002 Biochemical diagnosis of pheochromocytoma: which test is best? Journal of the American Medical Association 287 1427-1434.

Li J, Zhang Z, Rosenzweig J, Wang YY \& Chan DW 2002 Proteomics and bioinformatics approaches for identification of serum biomarkers to detect breast cancer. Clinical Chemistry 48 1296-1304.

Linnoila RI, Keiser HR, Steinberg SM \& Lack EE 1990 Histopathology of benign versus malignant sympathoadrenal paragangliomas: clinicopathologic study of 120 cases including unusual histologic features. Human Pathology 21 1168-1180.

Liotta LA, Ferrari M \& Petricoin E 2003 Clinical proteomics: written in blood. Nature $\mathbf{4 2 5} 905$.

Mehta AI, Ross S, Lowenthal MS, Fusaro V, Fishman DA, Petricoin EF 3rd \& Liotta LA 2003 Biomarker amplification by serum carrier protein binding. Disease Markers 19 1-10.

O'Riordain DS, Young WF Jr, Grant CS, Carney JA \& van Heerden JA 1996 Clinical spectrum and outcome of functional extraadrenal paraganglioma. World Journal of Surgery 20 916-921.

Orlando C \& Gelmini S 2001 Telomerase in endocrine and endocrine-dependent tumors. Journal of Steroid Biochemistry and Molecular Biology $\mathbf{7 8}$ 201-214.

Ornstein DK, Rayford W, Fusaro VA, Conrads TP, Ross SJ, Hitt BA, Wiggins WW, Veenstra TD, Liotta LA \& Petricoin EF 3rd 2004 Serum proteomic profiling can discriminate prostate cancer from benign prostates in men with total prostate specific antigen levels between 2.5 and $15.0 \mathrm{ng} / \mathrm{ml}$. Journal of Urology 172 1302-1305.

Pacak K, Linehan WM, Eisenhofer G, Walther MM \& Goldstein DS 2001 $a$ Recent advances in genetics, diagnosis, localization, and treatment of pheochromocytoma. Annals of Internal Medicine $\mathbf{1 3 4}$ 315-329. 
Pacak K, Chrousos GP, Koch CA, Lenders JW \& Eisenhofer G $2001 b$ Pheochromocytoma: progress in diagnosis, therapy, and genetics. In Adrenal Disorders, edn 1, pp 479-523. Eds A Margioris \& GP Chrousos. Totowa: Humana Press.

Petricoin EF, Ardekani AM, Hitt BA, Levine PJ, Fusaro VA, Steinberg SM, Mills GB, Simone C, Fishman DA, Kohn EC et al. $2002 a$ Use of proteomic patterns in serum to identify ovarian cancer. Lancet 359 572-577.

Petricoin EF 3rd, Ornstein DK, Paweletz CP, Ardekani A, Hackett PS, Hitt BA, Velassco A, Trucco C, Wiegand L, Wood K et al. 2002 b Serum proteomic patterns for detection of prostate cancer. Journal of the National Cancer Institute 94 1576-1578.

Qu Y, Adam BL, Yasui Y, Ward MD, Cazares LH, Schellhammer PF, Feng Z, Semmes OJ \& Wright GL Jr 2002 Boosted decision tree analysis of surface-enhanced laser desorption/ionization mass spectral serum profiles discriminates prostate cancer from noncancer patients. Clinical Chemistry 48 1835-1843.

Rao F, Keiser HR \& O'Connor DT 2002 Malignant and benign pheochromocytoma: chromaffin granule transmitters and the response to medical and surgical treatment. Annals of the New York Academy of Sciences $971530-532$.
Rui Z, Jian-Guo J, Yuan-Peng T, Hai P \& Bing-Gen R 2003 Use of serological proteomic methods to find biomarkers associated with breast cancer. Proteomics $\mathbf{3}$ 433-439.

Sinz A, Bantscheff M, Mikkat S, Ringel B, Drynda S, Kekow J, Thiesen HJ \& Glocker MO 2002 Mass spectrometric proteome analyses of synovial fluids and plasmas from patients suffering from rheumatoid arthritis and comparison to reactive arthritis or osteoarthritis. Electrophoresis 23 3445-3456.

Tanaka S, Ito T, Tomoda J, Higashi T, Yamada G \& Tsuji T 1993 Malignant pheochromocytoma with hepatic metastasis diagnosed 20 years after resection of the primary adrenal lesion. Internal Medicine 32 789-794.

Vytvytska O, Nagy E, Bluggel M, Meyer HE, Kurzbauer R, Huber LA \& Klade CS 2002 Identification of vaccine candidate antigens of Staphylococcus aureus by serological proteome analysis. Proteomics 2 580-590.

Wadsworth JT, Somers KD, Cazares LH, Malik G, Adam BL, Stack BC Jr, Wright GL Jr \& Semmes OJ 2004 Serum protein profiles to identify head and neck cancer. Clinical Cancer Research 10 1625-1632.

Wulfkuhle JD, Liotta LA \& Petricoin EF 2003 Proteomic applications for the early detection of cancer. Nature Reviews Cancer 3 267-275. 